\title{
CLASSIFICATION OF ALL HEREDITARILY INDECOMPOSABLE CIRCULARLY CHAINABLE CONTINUA $\left({ }^{1}\right)$
}

BY

\author{
LAWRENCE FEARNLEY $\left({ }^{2}\right)$
}

\begin{abstract}
In a recent paper the author has established an affirmative solution to a well-known and previously unsolved problem raised by R. H. Bing in 1951 concerning whether or not the pseudo-circle is topologically unique. Now in this present paper, as the natural culmination of the study initiated in the earlier paper, complete topological and mapping classification theorems are established for all hereditarily indecomposable circularly chainable continua. The principal topological classification results of this paper are the theorems that hereditarily indecomposable circularly chainable continua are characterized set-theoretically by their equivalence classes of fundamental sequences and are characterized algebraic-topologically by their Cech cohomology groups. These topological classification theorems are then used in establishing mapping classification theorems for all hereditarily indecomposable circularly chainable continua and in proving that the mapping hierarchy of hereditarily indecomposable circularly chainable continua constitutes a lattice. Among the consequences of the foregoing primary results of the paper are the additional theorems that two hereditarily indecomposable circularly chainable continua are topologically equivalent if and only if each of them is a continuous image of the other, and that every $k$-adic pseudo-solenoid is topologically unique.
\end{abstract}

1. Introduction. In a recent paper in this journal [10], a research announcement of which was published in [8], this author has given an affirmative solution to a well-known problem concerning limit spaces originally raised by $\mathrm{R}$. H. Bing in 1951 in [3, p. 49], regarding whether or not all pseudo-circles are topologically equivalent. Now, in this present paper, as the natural culmination of the study initiated in the above paper of Fearnley [10], complete topological and mapping classifications will be established for all hereditarily indecomposable circularly chainable continua.

First, in $\S 3$, two topological classifications of all hereditarily indecomposable circularly chainable continua are established in terms of set-theoretic and algebraic topological characteristics, respectively. Then these topological classifications are

Presented to the Society, October 9, 1969; received by the editors August 18, 1970.

AMS 1970 subject classifications. Primary 54F15, 54F65.

Key words and phrases. Hereditarily indecomposable continua, circularly chainable continua, classification of pseudo-solenoids, mapping hierarchy lattice.

( ${ }^{1}$ ) This research was supported by the National Science Foundation under grant NSFGP6308.

$\left({ }^{2}\right)$ The author wishes to thank Professor R. H. Bing for his helpful advice which contributed to this research. 
used in proving the uniqueness of certain major subclasses of the class of all hereditarily indecomposable circularly chainable continua. Among the results established in this category is the theorem that all $k$-adic pseudo-solenoids are topologically unique.

Next, in $\S 4$, necessary and sufficient conditions are established for the determination of whether or not an arbitrary hereditarily indecomposable circularly chainable continuum is a continuous image of another continuum in this category of continua. This mapping classification of all hereditarily indecomposable circularly chainable continua is then used in the development of a hierarchical lattice of all generalized pseudo-circles.

Finally, in $\S 4$, consequences of the combination of the topological classifications of all hereditarily indecomposable circularly chainable continua established in $\$ 3$ and the mapping classification of all hereditarily indecomposable circularly chainable continua established in $\S 4$ are developed. In particular, necessary and sufficient conditions are established in terms of the mapping characteristics of two arbitrary hereditarily indecomposable circularly chainable continua $L$ and $M$ in order for $L$ and $M$ to be homeomorphic.

2. Preliminaries. The special terms and notations used in this paper will be primarily the same as those introduced by this author in [6], [7], [9] and [10]. In general these terms and notations were suggested by those used by Bing in [2], [3] and [4]. In addition we shall define a number of further terms and notations which will be of particular use in the development of the classification theorems of this paper.

First let $M$ be a circularly chainable continuum and let $C_{1}, C_{2}, C_{3}, \ldots$ and $f_{1}, f_{2}, f_{3}, \ldots$ be sequences of circular chains and cyclic $r$-patterns, respectively, having the following properties:

(a) Each circular chain $C_{i}$ of the sequence $C_{1}, C_{2}, C_{3}, \ldots$ covers $M$ and the closures of nonadjacent links of $C_{i}$ are mutually exclusive.

(b) The mesh of $C_{i}, i=1,2,3, \ldots$, approaches zero as $i$ increases without bound.

(c) The pattern $f_{i}, i=1,2,3, \ldots$, is a cyclic $r$-pattern of $C_{i+1}$ in $C_{i}$ such that the winding number $w\left(f_{i}\right)$ of $f_{i}$ is greater than 1 .

(d) The closure of each link of $C_{i+1}$ is a subset of the link of $C_{i}$ to which it corresponds under $f_{i}, i=1,2,3, \ldots$.

The system of circular chains $C_{1}, C_{2}, C_{3}, \ldots$ and cyclic $r$-patterns $f_{1}, f_{2}, f_{3}, \ldots$ will be said to be multicyclically associated with $M$ and will be denoted by $\left(C_{1}, f_{1}, C_{2}, f_{2}, C_{3}, f_{3}, \ldots\right)$. The term "multicyclically associated" is chosen to distinguish the properties (a), (b), (c) and (d), above, from those described in the definition of "cyclically associated" in [7] where it was required that all cyclic $r$-patterns considered be monocyclic $r$-patterns.

Next let $\left(C_{1}, f_{1}, C_{2}, f_{2}, C_{3}, f_{3}, \ldots\right)$ be a system of circular chains and cyclic $r$-patterns multicyclically associated with a circularly chainable continuum $M$ and, 
for each positive integer $i$, let the winding number $w\left(f_{i}\right)$ of $f_{i}$ be represented as a product of primes by a relationship of the form

$$
w\left(f_{i}\right)=x_{i 1} x_{i 2} \cdots x_{i n_{i}} .
$$

Then we define the sequence of primes

$$
x_{11}, x_{12}, \ldots, x_{1 n_{1}}, x_{21}, x_{22}, \ldots, x_{2 n_{2}}, x_{31}, x_{32}, \ldots, x_{3 n_{3}}, \ldots
$$

to be a fundamental sequence of $M$. This sequence of primes will also be referred to as a prime trace of the system $\left(C_{1}, f_{1}, C_{2}, f_{2}, C_{3}, f_{3}, \ldots\right)$ or as a prime trace of the sequence of cyclic r-patterns $f_{1}, f_{2}, f_{3}, \ldots$

An hereditarily indecomposable circularly chainable continuum which has the same Čech cohomology characteristics as a $k$-adic solenoid is called a $k$-adic pseudo-solenoid.

A generalized pseudo-circle is a nonsnakelike hereditarily indecomposable circularly chainable continuum. The term pseudo-circle without modifying adjective is reserved as in the usage of Jones [13] to identify the original nonsnakelike planar hereditarily indecomposable circularly chainable continuum constructed by Bing [3] and shown to be topologically unique by Fearnley [10].

The foregoing definitions are applicable in the study of nonplanar circularly chainable continua. For the case of snakelike circularly chainable continua, certain categories of which have been discussed by Burgess [5], associated systems of circular chains and cyclic $r$-patterns can be chosen in which all cyclic $r$-patterns have winding numbers equal to zero. For the case of nonsnakelike planar circularly chainable continua, it is a consequence of the results of [4] that associated systems of circular chains and cyclic $r$-patterns can be chosen in which all of the cyclic $r$-patterns have winding numbers equal to 1 . Thus we define the fundamental sequence of a snakelike circularly chainable continuum to be the sequence $0,0,0, \ldots$ and we define the fundamental sequence of a nonsnakelike planar circularly chainable continuum to be the sequence $1,1,1, \ldots$. The pseudo-arc can be considered as a 0 -adic pseudo-solenoid and the pseudo-circle can be considered as a 1-adic pseudosolenoid.

Now let $N$ denote the set of all natural numbers. The group of definitions which follow involves certain finite and infinite sequences of elements of $N$. If $X=$ $\left(x_{1}, x_{2}, x_{3}, \ldots\right)$ is an infinite sequence of elements of $N$ and $k$ is a positive integer then the subsequence $\left(x_{k}, x_{k+1}, x_{k+2}, \ldots\right)$ of $X$ is said to be a last section of $X$. The ordered sum of a finite sequence $X=\left(x_{1}, x_{2}, \ldots, x_{n}\right)$ of elements of $N$ and a finite or infinite sequence $Y=\left(y_{1}, y_{2}, \ldots, y_{m}\right)$ or $Y=\left(y_{1}, y_{2}, y_{3}, \ldots\right)$, respectively, of elements of $N$ is the sequence $\left(x_{1}, x_{2}, \ldots, x_{n}, y_{1}, y_{2}, \ldots, y_{m}\right)$ or $\left(x_{1}, x_{2}, \ldots, x_{n}\right.$, $\left.y_{1}, y_{2}, y_{3}, \ldots\right)$, respectively, obtained by the juxtaposition of $X$ and $Y$. The notation $\{X\}+\{Y\}$ will be used to indicate this ordered sum of $X$ and $Y$. If $X=\left(x_{1}, x_{2}, \ldots, x_{n}\right)$ is a finite sequence of elements of $N$, the product $x_{1} x_{2} \cdots x_{n}$ will be referred to as the product of $X$ and will be denoted by $\Pi\{X\}$.

Next let $p$ be an arbitrary element of $N$ and let $X$ be a sequence of elements of $N$. 
Then the cardinal number corresponding to the number of occurrences of $p$ in $X$ will be defined to be the multiplicity of $p$ in $X$ and will be indicated by the notation $m(p, X)$. A sequence $C$ is said to be a complement of a finite sequence $Y$ of elements of $N$ relative to a finite or infinite sequence $X$ of elements of $N$ if, for each element $p$ of $N, m(p, C)=m(p, X)-m(p, Y)$. A union $U$ of two finite or infinite sequences $X$ and $Y$ of elements of $N$ is a sequence such that, for each element $p$ of $N$,

$$
m(p, U)=\operatorname{maximum}(m(p, X), m(p, Y)) \text {. }
$$

An intersection $I$ of two finite or infinite sequences $X$ and $Y$ of elements of $N$ is a sequence such that, for each element $p$ of $N$,

$$
m(p, I)=\operatorname{minimum}(m(p, X), m(p, Y)) \text {. }
$$

While the complement, union and intersection sequences described in the preceding definitions are not uniquely determined sequences, it will be convenient, where no confusion is likely to arise, to indicate sequences of the types $C, U$ and $I$ by the notations $\{X\}-\{Y\},\{X\} \cup\{Y\}$ and $\{X\} \cap\{Y\}$, respectively.

If $X$ and $Y$ are finite or infinite sequences of elements of $N$ such that, for each element $p$ of $N, m(p, X) \leqq m(p, Y)$ then the sequence $X$ is said to be contained in the sequence $Y$. This relationship will be denoted by $\{X\} \subset\{Y\}$. The joint relationships $\{X\} \subset\{Y\}$ and $\{Y\} \subset\{X\}$ between finite or infinite sequences $X$ and $Y$ of elements of $N$ will then be indicated by the notation $\{X\} \approx\{Y\}$. Two finite or infinite sequences $X$ and $Y$ of points of $N$ will be defined to be equipotent if $\{X\} \approx\{Y\}$.

Two fundamental sequences associated with circularly chainable continua will be defined to be equivalent if these fundamental sequences are equipotent sequences of natural numbers.

Finally, to facilitate the presentation of arguments involving pairs of systems of circular chains and cyclic $r$-patterns associated with circularly chainable continua it will be convenient to extend the usage of the concept of cyclic $r$-pattern to include the situation in which a cyclic $r$-pattern $f$ has the sets of subscripts of circular chains $C$ and $D$ as domain and range, respectively, whether or not there exist containment relationships between the links of $D$ and the links of $C$. Such a cyclic $r$-pattern will be referred to as a cyclic r-pattern from $C$ to $D$ and will be denoted by $f: C \rightarrow D$ or $C \stackrel{f}{\rightarrow} D$.

It will be assumed throughout this paper that all circularly chainable continua considered are nondegenerate topological spaces and that all circular chains considered have at least six links. The latter assumption will allow us to use the techniques of earlier related papers of this author and to avoid the formal awkwardness of considering situations where the crookedness properties or winding characteristics are trivial. It will be seen that these assumptions can be made without any loss in generality in the results of this paper.

3. Topological classification of all hereditarily indecomposable circularly chainable continua. The purpose of this section is to establish two of the three principal 
topological classification theorems of this paper. In particular we shall prove that hereditarily indecomposable circularly chainable continua are characterized topologically by their equivalence classes of fundamental sequences and by their Čech cohomology properties. A third topological characterization of this class of continua will be presented in $\$ 4$.

First we establish an important preliminary theorem that is a generalization of [10, Theorem 6.1] and a partial generalization of [8, Theorem 2], the results of which were established previously by this author.

THEOREM 3.1. Let $M$ be a nonsnakelike circularly chainable continuum, let $\left(C_{1}, f_{1}, C_{2}, f_{2}, C_{3}, f_{3}, \ldots\right)$ be a system of circular chains and cyclic r-patterns multicyclically associated with $M$, and let $D$ be a circular p-chain such that $D$ is a principal refinement of $C_{1}$ with the property that the cyclic r-pattern $f$ of $D$ in $C_{1}$ has positive winding number. In addition, for each positive integer $i$, let $f_{i}$ be a crooked cyclic $r$-pattern. Then, if there is an integer $m$ such that the winding number of $f$ divides the winding number of the composite cyclic r-pattern $f_{1} f_{2} \cdots f_{m}$, there exists an integer $n$ greater than $m$ and a cyclic r-pattern $t$ such that $f t=f_{1} f_{2} \cdots f_{n}$.

Proof. Since each cyclic $r$-pattern of the sequence $f_{1}, f_{2}, f_{3}, \ldots$ has positive winding number, we may assume, noting [7, Lemma 3.3.2] and the proof of [7, Theorem 3.3], that each cyclic $r$-pattern $f_{i}$ of the sequence $f_{1}, f_{2}, f_{3}, \ldots$ has the properties that $f_{i}(0)=0$ and $f_{i}$ is properly left-normal. In addition, from conditions (a), (b) and (d) of the definition of "multicyclically associated", it may also be assumed without loss in generality that each cyclic $r$-pattern $f_{i}$ of the sequence $f_{1}, f_{2}, f_{3}, \ldots$ has rank greater than or equal to 2 . In addition, for each positive integer $i$ the cyclic $r$-pattern $f_{i}$ is crooked so that it follows by [10, Theorem 3.1] that $f_{i}$ can be assumed to have the property that the length of the canonical linear representation of $f_{i}$ is greater than or equal to twice the length of $f_{i}$.

Now there is an integer $m$ such that the winding number of $f$ divides the winding number of the composite cyclic $r$-pattern $f_{1} f_{2} \cdots f_{m}$. Thus, we may follow an argument similar to that given in the proof of [10, Theorem 5.1] to obtain an integer $n$ greater than $m$ and a cyclic $r$-pattern $t$ such that $f t=f_{1} f_{2} \cdots f_{n}$. This completes the proof.

It is observed that actually a stronger form of Theorem 3.1 can be established which is a complete generalization of [8, Theorem 2] and which, in particular, includes a uniformity conclusion similar to that of [8, Theorem 2]. Specifically a similar argument to that given in Theorem 3.1 can be used to show that if the additional condition is added to those conditions listed in the definition of "multicyclically associated" that requires that all cyclic $r$-patterns considered shall have rank greater than or equal to 2 then the integer $n$ can be chosen uniformly in the sense that the difference $n-m$ depends only on $f$ and not on the particular nature of the cyclic $r$-patterns $f_{m+1}, f_{m+2}, f_{m+3}, \ldots$ 
We now establish the first of the principal topological classification theorems of this paper.

THEOREM 3.2. In order that two hereditarily indecomposable circularly chainable continua $L$ and $M$ be homeomorphic it is necessary and sufficient that $L$ and $M$ have equivalent fundamental sequences.

Proof of necessity. Let $L$ and $M$ be homeomorphic and let $h$ be a homeomorphism of $L$ onto $M$. It will be assumed that $L$ and $M$ are nonplanar hereditarily indecomposable circularly chainable continua since it has been shown by Bing [3] that any two snakelike hereditarily indecomposable continua are topologically equivalent and by Fearnley [10] that any two nonsnakelike planar hereditarily indecomposable circularly chainable continua are also topologically equivalent. Then there exist systems of circular chains and cyclic $r$-patterns

$$
\left(C_{1}, f_{1}, C_{2}, f_{2}, C_{3}, f_{3}, \ldots\right) \text { and }\left(D_{1}, g_{1}, D_{2}, g_{2}, D_{3}, g_{3}, \ldots\right)
$$

which are multicyclically associated with $L$ and $M$, respectively. The notations $h\left(C_{i}\right)$ and $h^{-1}\left(D_{j}\right)$, where $i$ and $j$ are positive integers, will be used to denote the circular chains covering $M$ and $L$, respectively, whose links are the images under the respective homeomorphisms $h$ and $h^{-1}$ of the links of $C_{i}$ and $D_{j}$, respectively.

Now $h$ is a homeomorphism with the compact domain $L$ so that $h$ is uniformly continuous. In addition the mesh of $C_{i}$ approaches zero as $i$ increases without bound. Furthermore, since $M$ is a compact continuum, each of the coverings $D_{1}, D_{2}, D_{3}, \ldots$ of $M$ has the Lebesgue number property. Thus there is an integer $n_{1}$ such that $h\left(C_{n_{1}}\right)$ is a refinement of $D_{1}$ with the property that the closure of each link of $h\left(C_{n_{1}}\right)$ is contained in the link of $D_{1}$ to which it corresponds under the $r$-pattern of $h\left(C_{n_{1}}\right)$ in $D_{1}$. We may assume that the cyclic $r$-pattern of $h\left(C_{n_{1}}\right)$ in $D_{1}$ has positive winding number. Next, since the mesh of $D_{i}$ approaches zero as $i$ increases without bound and each of the open coverings $h\left(C_{1}\right), h\left(C_{2}\right), h\left(C_{3}\right), \ldots$ of the compact continuum $M$ has the Lebesgue number property, there is an integer $m_{1}$ greater than 1 such that $D_{m_{1}}$ is a refinement of $h\left(C_{n_{1}}\right)$. In a similar manner we may choose increasing sequences of positive integers $n_{1}, n_{2}, n_{3}, \ldots$ and $m_{1}, m_{2}, m_{3}, \ldots$ such that, for each positive integer $i, h\left(C_{n_{t+1}}\right)$ is a refinement of $D_{m_{i}}$ and $D_{m_{i+1}}$ is a refinement of $h\left(C_{n_{i+1}}\right)$.

Hence there is a commutative diagram of the following form in which $h^{*}$ indicates the cyclic $r$-pattern induced by $h$ and the arrows indicate surjective cyclic $r$-patterns under the convention described in $\S 2$.

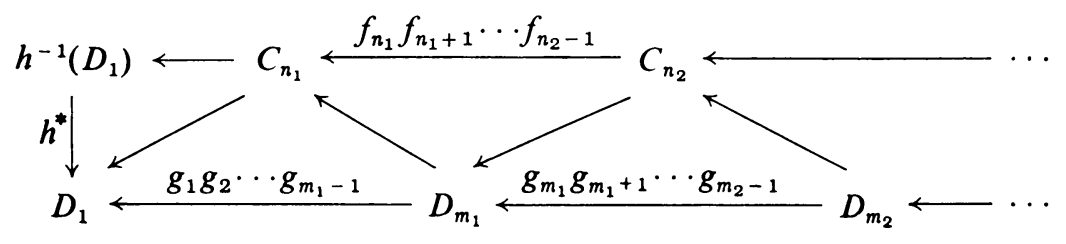


Then, noting the properties of the cyclic $r$-pattern of $C_{n_{1}}$ in $h^{-1}\left(D_{1}\right)$, the circular chains and cyclic $r$-patterns identified on the upper of the two horizontal levels of the diagram constitute a system which is multicyclically associated with $L$. In addition, the circular chains and cyclic $r$-patterns identified on the lower of the two horizontal levels of the diagram constitute a system multicyclically associated with $M$. Hence, by [7, Theorem 3.2] together with the facts that $h^{*}$ has winding number 1 and that the diagram is commutative, we conclude that a fundamental sequence of $L$ obtained by a decomposition into primes of the winding numbers of the cyclic $r$-patterns of the upper horizontal level of the diagram is a permutation of a fundamental sequence of $M$ obtained in a similar manner from the cyclic $r$-patterns of the lower horizontal level of the diagram. Therefore, $L$ and $M$ have equivalent fundamental sequences.

Proof of sufficiency. Let $L$ and $M$ have equivalent fundamental sequences $X=\left(x_{1}, x_{2}, x_{3}, \ldots\right)$ and $Y=\left(y_{1}, y_{2}, y_{3}, \ldots\right)$. It will be assumed that each member of each of the sequences $X$ and $Y$ is greater than 1 in view of the result of Bing [3] that any two snakelike hereditarily indecomposable continua are homeomorphic and the result of Fearnley [10] that any two nonsnakelike planar hereditarily indecomposable circularly chainable continua are also topologically equivalent. Then there are systems of circular chains and cyclic $r$-patterns

$$
\left(C_{1}, f_{1}, C_{2}, f_{2}, C_{3}, f_{3}, \ldots\right) \text { and }\left(D_{1}, g_{1}, D_{2}, g_{2}, D_{3}, g_{3}, \ldots\right)
$$

which are multicyclically associated with $L$ and $M$, respectively, and which have $X$ and $Y$, respectively, as prime traces. In addition $L$ and $M$ are hereditarily indecomposable circularly chainable continua so that, without loss in generality, we may assume that each cyclic $r$-pattern of each of the sequences $f_{1}, f_{2}, f_{3}, \ldots$ and $g_{1}, g_{2}, g_{3}, \ldots$ is a crooked cyclic $r$-pattern. Furthermore if one of the circular chains $C_{1}$ and $D_{1}$ has more links than the other then links of this circular chain can be combined and the cyclic $r$-pattern having this circular chain as range can be correspondingly modified, so that we may assume that $C_{1}$ and $D_{1}$ have been chosen to have equal numbers of links.

Now let $e$ denote the identity cyclic $r$-pattern from $C_{1}$ to $D_{1}$. Then, by Theorem 3.1 , there is an integer $v_{1}$ and a cyclic $r$-pattern $t_{1}$ such that $e t_{1}=g_{1} g_{2} \cdots g_{v_{1}-1}$. Furthermore, noting [7, Theorem 3.2], the winding number $w\left(t_{1}\right)$ of $t_{1}$ is equal to the product of the winding numbers of $g_{1}, g_{2}, \ldots, g_{v_{1}-1}$. We denote by $Y_{v_{1}}$ a finite sequence of primes whose product is equal to $w\left(t_{1}\right)$. Then $\left\{Y_{v_{1}}\right\} \subset\{Y\} \approx\{X\}$. Hence, again using Theorem 3.1, there is an integer $u_{1}$ and a cyclic $r$-pattern $t_{2}$ such that $t_{1} t_{2}=f_{1} f_{2} \cdots f_{u_{1}-1}$. We denote by $X_{u_{1}}$ a finite sequence of primes whose product is equal to the winding number $w\left(t_{2}\right)$ of $t_{2}$. It follows that $\left\{X_{u_{1}}\right\} \subset\left\{X-Y_{v_{1}}\right\} \approx\left\{Y-Y_{v_{1}}\right\}$. Thus we may again apply Theorem 3.1 to obtain an integer $v_{2}$ and a cyclic $r$-pattern $t_{3}$ such that $t_{2} t_{3}=g_{v_{1}} g_{v_{1}+1} \cdots g_{v_{2}-1}$. Proceeding in this manner we obtain a commutative diagram of circular chains and surjective cyclic $r$-patterns with the following form under the convention of $\S 2$. 


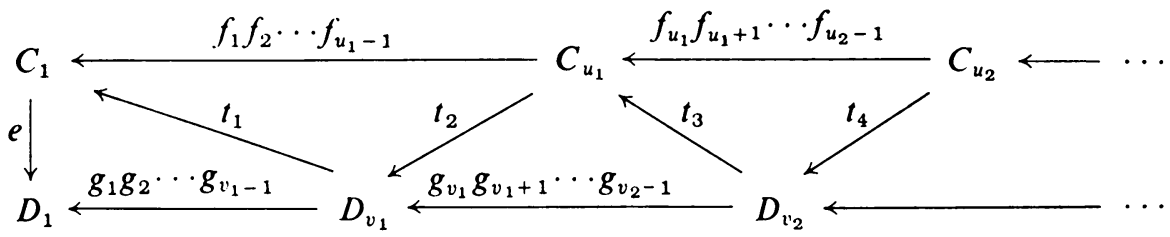

Therefore, from the relationship of this diagram to a corresponding inverse limit diagram as described in $[10, \S 6]$ and from the properties of the resulting system [1] or [14], we conclude that $L$ and $M$ are topologically equivalent.

The theorem which follows constitutes an algebraic-topological classification of all hereditarily indecomposable circularly chainable continua which will be used subsequently in establishing the uniqueness of each of the pseudo-solenoids. This algebraic-topological classification is surprisingly elegant in view of the local complexity [3] and global nonhomogeneity [9] of nonsnakelike hereditarily indecomposable circularly chainable continua.

THEOREM 3.3. In order that two hereditarily indecomposable circularly chainable continua $L$ and $M$ be homeomorphic it is necessary and sufficient that the onedimensional Čech cohomology groups of $L$ and $M$ be isomorphic.

Proof. In view of the topological invariance of the Čech cohomology groups it will be sufficient to consider the situation in which $L$ and $M$ are not homeomorphic and prove that then the one-dimensional Čech cohomology groups $H^{1}(L)$ and $H^{1}(M)$ of $L$ and $M$, respectively, are not isomorphic. In addition, as in the case of Theorem 3.2, the desired result for snakelike hereditarily indecomposable circularly chainable continua follows from the results of Bing [3] and the result for nonsnakelike planar hereditarily indecomposable circularly chainable continua is a consequence of the results of Fearnley [10] so that we shall assume that $L$ and $M$ are nonplanar hereditarily indecomposable circularly chainable continua.

With the foregoing assumptions we show now that there exist two fundamental sequences $X$ and $Y$ of $L$ and $M$, respectively, such that either no last section of $X$ is contained in $Y$ or no last section of $Y$ is contained in $X$. Suppose that there do not exist such fundamental sequences and let $A$ and $B$ be any two fundamental sequences of $L$ and $M$, respectively. Then we may choose a last section $A_{1}$ of $A$ which is a fundamental sequence of $L$ and a last section $B_{1}$ of $B$ such that $\left\{B_{1}\right\} \subset\left\{A_{1}\right\} \subset\{B\}$. Furthermore, by Theorem 3.2 together with the fact that $L$ and $M$ are not homeomorphic, both of the inclusion relationships of the preceding expression are proper inclusions. Then, for each prime $p$ which is not a member of $B-B_{1}, m\left(p, A_{1}\right)$ $=m(p, B)$. In addition $B-A_{1}$ is a nonempty finite sequence. Thus, if $\left(C_{1}, f_{1}, C_{2}, f_{2}\right.$, $\left.C_{3}, f_{3}, \ldots\right)$ is a system of circular chains and cyclic $r$-patterns having a prime trace $A_{1}$ and being multicyclically associated with $L$, there is a circular chain $C$ and a cyclic $r$-pattern $f$ of $C_{1}$ in $C$ such that the winding number of $f$ is equal to $\prod\left\{B-A_{1}\right\}$. Therefore, the system $\left(C_{1}, f_{1}, C_{2}, f_{2}, C_{3}, f_{3}, \ldots\right)$ is multicyclically associated with $L$ 
and has a prime trace equivalent to $B$ which involves a contradiction to Theorem 3.2.

Now let $X$ and $Y$ be fundamental sequences of $L$ and $M$, respectively, such that no last section of $X$ is contained in $Y$ and let

$$
\left(C_{1}, f_{1}, C_{2}, f_{2}, C_{3}, f_{3}, \ldots\right) \text { and }\left(D_{1}, g_{1}, D_{2}, g_{2}, D_{3}, g_{3}, \ldots\right)
$$

be systems of circular chains and cyclic $r$-patterns multicyclically associated with $L$ and $M$, respectively, having $X$ and $Y$ as prime traces, respectively. Next, noting the argument given in [11, Theorem 5.2], we may assume that $H^{1}(L)$ is the additive group of all rational numbers of the form $u / d_{1} d_{2} \cdots d_{r}$, where $d_{i}$ is the winding number of $f_{i}, i=1,2, \ldots, r, r=1,2,3, \ldots$, and that $H^{1}(M)$ is the additive group of all rational numbers of the form $v / e_{1} e_{2} \cdots e_{s}$, where $e_{j}$ is the winding number of $g_{j}, j=1,2, \ldots, s, s=1,2,3, \ldots$ Suppose that there is an isomorphism $\theta$ of $H^{1}(L)$ onto $H^{1}(M)$ and let $\theta(1)=v_{1} / e_{1} e_{2} \cdots e_{s_{1}}$. We choose $A$ to be a finite sequence of prime integers whose product is equal to $v_{1}$. Then $X$ can be expressed as an ordered sum $\{U\}+\{V\}$ such that $\{A\} \cap\{X\} \subset\{U\}$. Hence, since no last section of $X$ is contained in $Y$, there is a prime $p$ such that $m(p, V)>m(p, Y)$. Thus $V$ can be expressed as an ordered sum $\left\{V_{1}\right\}+\left\{V_{2}\right\}$ such that $m\left(p, V_{1}\right)>m(p, Y)$ and such that there is an integer $z$ with the property that $\prod\left\{\{U\}+\left\{V_{1}\right\}\right\}=d_{1} d_{2} \cdots d_{z}$. In addition, since $\theta$ is an isomorphism,

$$
\theta\left(1 / d_{1} d_{2} \cdots d_{z}\right)=\theta(1) / d_{1} d_{2} \cdots d_{z}=v_{1} / e_{1} e_{2} \cdots e_{s_{1}} d_{1} d_{2} \cdots d_{z}
$$

Therefore, from the characterizing properties of $U$ and $V_{1}$ we obtain the contradiction that this last expression does not represent any member of $H^{1}(M)$ and the proof is complete.

The following theorem, Theorem 3.4, is an immediate consequence of Theorem 3.3 and is a generalization of the principal result of [10] that the pseudo-circle is unique.

THEOREM 3.4. For each positive integer $k$, the $k$-adic pseudo-solenoid is topologically unique.

4. Mapping classification of all hereditarily indecomposable circularly chainable continua. In this section we establish necessary and sufficient conditions for an hereditarily indecomposable circularly chainable continuum $M$ to be a continuous image of another hereditarily indecomposable circularly chainable continuum $L$ in terms of the topological classifications of such continua developed in the preceding section. This result together with the topological classification results of $\S 3$ will then be used to prove that the mapping hierarchy of hereditarily indecomposable circularly chainable continua has the properties of a lattice. Findlly, the topological classification theorems of $\S 3$ and the mapping classification result of $\S 4$ will be used to establish a further topological classification of all hereditarily indecomposable circularly chainable continua. 
To facilitate the statement of the mapping classification theorem for all hereditarily indecomposable circularly chainable continua, we first define a relationship " $<$ " between ordered pairs of fundamental sequences in the following manner. A fundamental sequence $X$ is said to be infrapotent relative to a fundamental sequence $Y$, and this relationship is denoted by $\{X\}<\{Y\}$, if either

1. $X$ and $Y$ are sequences of prime integers such that $\{X\} \subset\{Y\}$,

2. $X$ is the fundamental sequence $(1,1,1, \ldots)$ and $Y$ is a sequence of prime integers, or

3. $X$ is the fundamental sequence $(0,0,0, \ldots)$.

In this situation it is sometimes convenient to refer to $Y$ as being ultrapotent relative to $X$ and this latter relationship is denoted by $\{Y\}>\{X\}$. We note that if $X$ and $Y$ are fundamental sequences then $X$ and $Y$ are equipotent if and only if $\{X\}<\{Y\}$ and $\{Y\}<\{X\}$.

THEOREM 4.1. In order that an hereditarily indecomposable circularly chainable continuum $M$ be a continuous image of an hereditarily indecomposable circularly chainable continuum $L$ it is necessary and sufficient that there exist fundamental sequences $X$ and $Y$ of $L$ and $M$, respectively, such that $X$ is ultrapotent relative to $Y$.

Proof of necessity. First we consider the case that $L$ and $M$ are nonplanar hereditarily indecomposable circularly chainable continua. Then let $t$ be a continuous transformation of $L$ onto $M$ and let

$$
\left(C_{1}, f_{1}, C_{2}, f_{2}, C_{3}, f_{3}, \ldots\right) \text { and }\left(D_{1}, g_{1}, D_{2}, g_{2}, D_{3}, g_{3}, \ldots\right)
$$

be systems of circular chains and cyclic $r$-patterns multicyclically associated with $L$ and $M$, respectively. Then, from the facts that $t$ is uniformly continuous that the mesh of $C_{i}$ approaches zero as $i$ increases without bound and that the open coverings $D_{1}, D_{2}, D_{3}, \ldots$ of $M$ have the Lebesgue number property, it follows that there exists an increasing sequence of positive integers $k_{1}, k_{2}, k_{3}, \ldots$ such that, for each positive integer $i$,

1. there is a cyclic $r$-pattern $t_{i}$ from $C_{k_{i}}$ to $D_{i}$ with the property that if $c_{j}$ is any link of $C_{k_{i}}$ then $t\left(c_{j}\right)$ is contained in the link of $D_{i}$ with subscript $t_{i}(j)$, and

2. each link of $D_{i}$ corresponds under $t_{i}$ to at least one link of $C_{k_{i}}$.

Hence there exists the following commutative diagram of circular chains and surjective cyclic $r$-patterns:

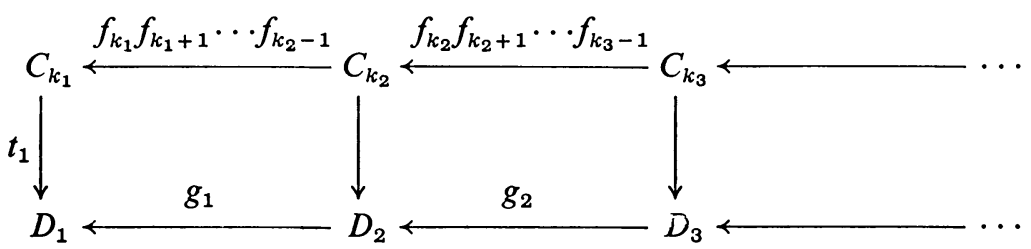


In addition there is a circular chain $E$ whose links are open sets of $L$ with the properties that $C_{k_{1}}$ has the cyclic $r$-pattern $t_{1}$ in $E$ and

$$
\left(E, t_{1}, C_{k_{1}}, f_{k_{1}} f_{k_{1}+1} \cdots f_{k_{2}-1}, C_{k_{2}}, f_{k_{2}} f_{k_{2}+1} \cdots f_{k_{3}-1}, \ldots\right)
$$

is multicyclically associated with $L$. We denote by $X$ a prime trace of this latter system and denote by $Y$ a prime trace of $\left(D_{1}, g_{1}, D_{2}, g_{2}, D_{3}, g_{3}, \ldots\right)$. Therefore, from the commutativity of the above diagram, $X$ contains $Y$ so that $\{X\}>\{Y\}$.

For the case that either $L$ or $M$ is a planar hereditarily indecomposable circularly chainable continuum the desired result follows from an argument similar to that given above which shows that no nonplanar generalized pseudo-circle is a continuous image of the pseudo-circle together with the results of [12] that neither the pseudo-circle nor any nonplanar generalized pseudo-circle is a continuous image of the pseudo-arc.

Proof of sufficiency. The case in which $Y$ is the pseudo-arc follows from [7, Theorem 4.4] of this author, and the case in which $Y$ is the pseudo-circle can be established by an argument similar to the one to be given for nonplanar generalized pseudo-circles. Thus we shall assume that $L$ and $M$ are nonplanar hereditarily indecomposable circularly chainable continua. Let $X$ and $Y$ be fundamental sequences of $L$ and $M$, respectively, such that $X$ contains $Y$ and let

$$
\left(C_{1}, f_{1}, C_{2}, f_{2}, C_{3}, f_{3}, \ldots\right) \text { and }\left(D_{1}, g_{1}, D_{2}, g_{2}, D_{3}, g_{3}, \ldots\right)
$$

be systems of circular chains and cyclic $r$-patterns multicyclically associated with $L$ and $M$, respectively, having prime traces $X$ and $Y$, respectively. Without loss in generality we may assume that $C_{1}$ and $D_{1}$ have equal numbers of links. In addition, since the circularly chainable continua $L$ and $M$ are hereditarily indecomposable, it may also be assumed that each cyclic $r$-pattern of each of the sequences $f_{1}, f_{2}$, $f_{3}, \ldots$ and $g_{1}, g_{2}, g_{3}, \ldots$ is a crooked cyclic $r$-pattern.

We now develop a modified and strengthened form of Theorem 3.1. First it is observed that Theorem 3.1 can be expressed solely in terms of $r$-patterns in the following manner. Let $r_{1}, r_{2}, r_{3}, \ldots$ be a sequence of crooked cyclic $r$-patterns such that, for each positive integer $i$, the range of $r_{i+1}$ is identical with the domain of $r_{1}$ and let $r$ be a cyclic $r$-pattern with the properties that the ranges of $r$ and $r_{1}$ are identical and for some positive integer $u$ the winding number of $r$ divides the winding number of the composite cyclic $r$-pattern $r_{1} r_{2} \cdots r_{u}$. Then there is a cyclic $r$-pattern $t$ and an integer $v$ greater than $u$ such that $r t=r_{1} r_{2} \cdots r_{v}$. We next strengthen this latter form of Theorem 3.1 using the fact that the method of proof of [10, Theorem 5.1] on which the proof of Theorem 3.1 depends may be followed even if, for some positive integer $k$, the cyclic $r$-patterns $r_{1}, r_{2}, \ldots, r_{k}$ are not crooked. Thus we obtain the result that if $r_{1}, r_{2}, r_{3}, \ldots$ is a sequence of cyclic $r$-patterns such that the range of $r_{i+1}$ is identical with the domain of $r_{i}, i=1,2$, $3, \ldots$, and all but at most finitely many members of the sequence $r_{1}, r_{2}, r_{3}, \ldots$ are crooked cyclic $r$-patterns and if $r$ is a cyclic $r$-pattern with the properties that the ranges of $r$ and $r_{1}$ are identical and, for some positive integer $u$, the winding 
number of $r$ divides the winding number of $r_{1} r_{2} \cdots r_{u}$, then there is a cyclic $r$-pattern $t$ and an integer $v$ greater than $u$ such that $r t=r_{1} r_{2} \cdots r_{v}$. This result will be used in the succeeding portion of the proof in establishing the existence of the desired continuous transformation of $L$ onto $M$.

To do this, first let $e$ denote the identity cyclic $r$-pattern from $C_{1}$ to $D_{1}$ and note that then the sequence of cyclic $r$-patterns $e, f_{1}, f_{2}, f_{3}, \ldots$ has the property that the range of each member of the sequence is identical with the domain of its predecessor, if any, in the sequence. In addition $X$ contains $Y$ and each of the cyclic $r$-patterns $f_{1}, f_{2}, f_{3}, \ldots$ is a crooked cyclic $r$-pattern. Hence, by the strengthened form of Theorem 3.1 developed in the preceding paragraph, there is a cyclic $r$-pattern $t_{1}$ and a positive integer $v_{1}$ such that $g_{1} t_{1}=e f_{1} f_{2} \cdots f_{v_{i}}$. Next, let $X_{1}, Y_{1}$ and $A_{1}$ be finite sequences whose products are equal to the winding numbers of $e f_{1} f_{2} \cdots f_{v_{1}}$, $g_{1}$ and $t_{1}$, respectively. Then, since $\{X\} \supset\{Y\}$, it follows that

$$
\left\{\left\{\{X\}-\left\{X_{1}\right\}\right\}+\left\{A_{1}\right\}\right\} \supset\left\{\{Y\}-\left\{Y_{1}\right\}\right\} \text {. }
$$

In addition each member of the sequence $f_{v_{1}+1}, f_{v_{1}+2}, f_{v_{1}+3}, \ldots$ is a crooked cyclic $r$-pattern whose range is identical with the domain of its predecessor in the augmented sequence $t_{1}, f_{v_{1}+1}, f_{v_{1}+2}, f_{v_{1}+3}, \ldots$. From the foregoing relationships and the strengthened form of Theorem 3.1 it follows that there is a cyclic $r$-pattern $t_{2}$ and an integer $v_{2}$ greater than $v_{1}$ such that $g_{2} t_{2}=t_{1} f_{v_{1}+1} f_{v_{1}+2} \cdots f_{v_{2}}$. Proceeding in this manner we obtain a commutative diagram of circular chains and surjective cyclic $r$-patterns having the following form:

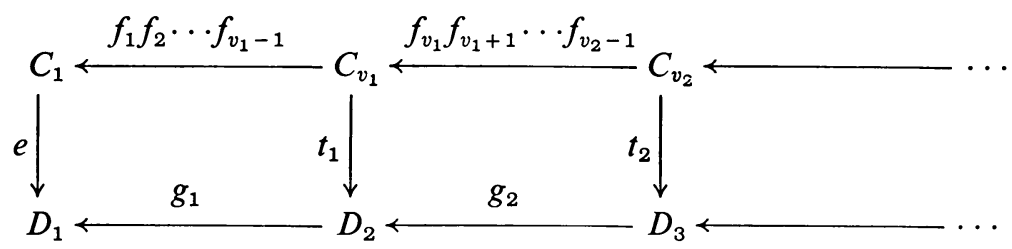

Therefore, from the relationship of this diagram to a corresponding inverse limit diagram as described in $[10, \S 6]$ and from the standard properties of the resulting system, we conclude that $M$ is a continuous image of $L$.

To facilitate the formulation of the next theorem two additional special terms will be defined. First let $\mathscr{M}$ be the class of all hereditarily indecomposable circularly chainable continua. A majorant $H$ of a pair $L, M$ of members of $\mathscr{M}$ is a member of $\mathscr{M}$ such that

1. the continua $L$ and $M$ are continuous images of $H$, and

2. for each continuum $K$ of the class $\mathscr{M}$ which can be mapped continuously onto each of the continua $L$ and $M$, the continuum $H$ is a continuous image of $K$.

The minorant of a pair $L, M$ of members of $\mathscr{M}$ is defined in a similar manner. In Theorem 4.2 it will be established that the mapping hierarchy of hereditarily indecomposable circularly chainable continua which is a consequence of Theorem 4.1 has the properties of a lattice in the following strong sense. 
THEOREM 4.2. Every pair of continua $L, M$ of the class $\mathscr{M}$ of all hereditarily indecomposable circularly chainable continua has a topologically unique majorant $H$ and a topologically unique minorant $K$.

Proof. Let $L$ and $M$ be nonplanar hereditarily indecomposable circularly chainable continua and let $X$ and $Y$ be fundamental sequences of $L$ and $M$, respectively. Then there exist hereditarily indecomposable circularly chainable continua $H$ and $K$ which have fundamental sequences $\{X\} \cup\{Y\}$ and $\{X\} \cap\{Y\}$, respectively. It will be proved that $H$ and $K$ are the topologically unique majorant and minorant, respectively, of the pair of continua $L, M$.

First, by Theorem 4.1 together with the fact that $\{X\} \cup\{Y\}$ contains each of $\{X\}$ and $\{Y\}$, the continuum $H$ can be mapped continuously onto each of $L$ and $M$. Next let $A$ be any hereditarily indecomposable circularly chainable continuum which can be mapped continuously onto each of $L$ and $M$. Then it follows from Theorem 4.1 that $A$ has fundamental sequences $U$ and $V$ that contain $X$ and $Y$, respectively. In addition, from the argument given in the third paragraph of the proof of Theorem 3.3 together with the fact that the one-dimensional Čech cohomology group representations of $A$ generated by $U$ and $V$ as described in the proof of Theorem 3.3 are isomorphic, there is a last section $U_{1}$ of $U$ contained in $V$. Furthermore, we may assume without loss in generality that $U_{1}$ has been chosen to be also a fundamental sequence of $A$. Hence we may again use the preceding method of argument to obtain the result that there is a last section $V_{1}$ of $V$ contained in $U_{1}$. Thus $\left\{V_{1}\right\} \subset\left\{U_{1}\right\} \subset\{V\}$. It follows that $\{V\}-\left\{U_{1}\right\}$ and $\{U\}-\left\{U_{1}\right\}$ are finite sequences. Thus it is easily seen that a sequence $U^{*}$ which satisfies the relationship

$$
U^{*} \approx\left\{V_{1}\right\} \cup\left\{\{V\}-\left\{U_{1}\right\}\right\} \cup\left\{\{U\}-\left\{U_{1}\right\}\right\}
$$

is a fundamental sequence of $A$ which contains each of $U$ and $V$ and hence contains $\{X\} \cup\{Y\}$. Therefore, by Theorem $4.1, H$ is a continuous image of $A$ and we conclude that $H$ is a majorant of the pair $L, M$.

To prove that $H$ is topologically unique suppose on the contrary that there exists an hereditarily indecomposable circularly chainable continuum $H^{*}$ such that $H^{*}$ is also a majorant of the pair $L, M$ but $H^{*}$ is not homeomorphic with $H$. Then, by Theorem 3.2, there do not exist fundamental sequences of $H$ and $H^{*}$ that are equivalent. Hence, by the argument given in the second paragraph of the proof of Theorem 3.3, there exist fundamental sequences $U$ and $U^{*}$ of $H$ and $H^{*}$, respectively, such that either no last section of $U$ is contained in $U^{*}$ or no last section of $U^{*}$ is contained in $U$. Since similar procedures of proof can be followed in both of these cases it will be assumed that no last section of $U$ is contained in $U^{*}$. Now $H$ and $H^{*}$ are majorants of the same pair $L, M$. Thus, in particular, the continuum $H$ is a continuous image of the continuum $H^{*}$. Hence, by Theorem 4.1, there are fundamental sequences $V$ and $V^{*}$ of $H$ and $H^{*}$, respectively, such that $V^{*}$ contains 
$V$. Now $U^{*}$ and $V^{*}$ are fundamental sequences of the same continuum $H^{*}$. Hence by an argument similar to that described in the preceding paragraph, there is a last section $V_{1}^{*}$ of $V^{*}$ such that $V_{1}^{*}$ is contained in $U^{*}$. Next we choose $V_{1}$ to be a last section of $V$ such that $V_{1}$ is contained in $V_{1}^{*}$ and note that we may assume without loss in generality that $V_{1}$ is a fundamental sequence of $H$. Hence we may again use a similar argument to obtain the result that there is a last section $U_{1}$ of $U$ such that $V_{1}$ contains $U_{1}$. Therefore, $\left\{U^{*}\right\} \supset\left\{V_{1}^{*}\right\} \supset\left\{V_{1}\right\} \supset\left\{U_{1}\right\}$ which involves a contradiction and we conclude that the majorant $H$ is topologically unique.

In a similar manner it can be shown that $K$ is a topologically unique minorant of the pair $L, M$.

Finally we note that in the case that either $L$ or $M$ is a planar hereditarily indecomposable circularly chainable continuum the desired result follows from the facts that the pseudo-arc is a continuous image of any generalized pseudo-circle, Fearnley [7, Theorem 4.4], that, by Theorem 4.1, the pseudo-circle is a continuous image of any nonplanar generalized pseudo-circle, and that the pseudo-arc and pseudo-circle are topologically unique ([3] and [10]). This completes the proof.

Finally we obtain a further topological classification of all hereditarily indecomposable circularly chainable continua with the following interesting form.

THEOREM 4.3. In order that two hereditarily indecomposable circularly chainable continua $L$ and $M$ be topologically equivalent it is necessary and sufficient that $M$ be a continuous image of $L$ and $L$ be a continuous image of $M$.

Proof. This is a corollary to the proof of Theorem 4.2.

\section{REFERENCES}

1. P. Alexandroff, Untersuchungen über Gestalt und Lage abgeschlossener Mengen beliebigen Dimension, Ann. of Math. 30 (1929), 101-187.

2. R. H. Bing, A homogeneous indecomposable plane continuum, Duke Math. J. 15 (1948), 729-742. MR 10, 261.

3. - Concerning hereditarily indecomposable continua, Pacific J. Math. 1 (1951), 43-51. MR 13, 265.

4. - Embedding circle-like continua in the plane, Canad. J. Math. 14 (1962), 113-128. MR 24 \#A1712.

5. C. E. Burgess, Chainable continua and indecomposability, Pacific J. Math. 9 (1959), 653-659. MR 22 \#1867.

6. L. Fearnley, Characterizations of the continuous images of the pseudo-arc, Trans. Amer. Math. Soc. 111 (1964), 380-399. MR 29 \#596.

7. - Characterization of the continuous images of all pseudo-circles, Pacific J. Math. 23 (1967), 491-513. MR 37 \#887.

8. —, The pseudo-circle is unique, Bull. Amer. Math. Soc. 75 (1969), 398-401. MR 39 \#7569.

9. - The pseudo-circle is not homogeneous, Bull. Amer. Math. Soc. 75 (1969), 554-558. MR 39 \#3460. 
10. L. Fearnley, The pseudo-circle is unique, Trans. Amer. Math. Soc. 149 (1970), 45-64. MR 41 \#6172.

11. - Embeddings of topological products of sphere-like continua, Proc. London Math. Soc. (3) 19 (1969), 586-600. MR 41 \#4494.

12. - Pseudo-circles and the pseudo-arc (to appear).

13. F. B. Jones, On homogeneity, Summary of Lectures and Seminars, The Summer Institute on Set-Theoretic Topology (Madison, Wis., 1955), Amer. Math. Soc., Providence, R. I., pp. 68-70.

14. J. Mioduszewski, Mappings of inverse limits, Colloq. Math. 10 (1963), 39-44. MR 29 \#4035.

Department of Mathematics, Brigham Young University, Provo, Utah 84601

Current address: School of Mathematics, Institute for Advanced Study, Princeton, New Jersey 08540 . 